\title{
Directing oscillatory transitions: Roles for noise and pulsing in global brain regulation
}

\author{
Denis Larrivee* \\ Loyola University Chicago, USA
}

\begin{abstract}
Most studies now indicate that mental representations are distributed, captured by non-linear neuronal dynamics that occur over widely spaced brain domains grounding global and regional activity. In the distributed and highly complex interconnectivity that characterizes global and local influences, attractors emerge from a high dimensional state space, shaped by multiple forces that elicit their physical realization in oscillatory rhythms. Such oscillations are computationally fruitful, linking sensory cues to cognitive operation and initiating activity trains that structure organismal responsivity. Mechanisms regulating oscillatory transitions entail deterministic and stochastic elements that function complementarily to access pervasive features of cognition, including spike timing dependency and entropic maxima, and that are constrained by the physical features of weak coupling and sparse connectivity in the state space.
\end{abstract}

\section{Introduction}

Most studies now indicate that mental representations are distributed, captured by neuronal dynamics that occur over widely spaced brain areas and grounded in functional mechanisms that process information at both global and regional levels [1]. Global function emerges from the local dynamics of each area while global dynamics in turn constrain localized activity, inducing a reciprocal and reciprocating influence that is mediated via recurrent interaction and ordered to self-organization [2].

Drawing from this paradigm, how global activity elicits and constrains local dynamics is related to the distributed configuration that is globally mediated and that is effected through each of its local modes of interaction. A significant example is the slaving principle where micronodes, or patterns of activity, become enslaved to the dominant and distributed state [3]. In such interaction the critical dimension is the mutual interactivity that is elicited from within the local dynamics. Increasingly, it is apparent that their mutual dependence is expressed through a dynamical non-linear architecture; hence, the form of the dependence explicitly arises from features that characterize the architecture of the dynamical model [4-6].

Fundamentally, dynamical models of cognition must account for organismal needs by linking constitutive operational features to properties of stability, flexibility, and integrity that are required for global performance. Models incorporating non-linear dynamics capture these properties theoretically. Stability, for example, is a constitutive feature of attractors, invariant mathematical solutions of dynamical systems to which the state of the neural system converges, or to which it returns when perturbed [7]. Attractors thus emerge from the dynamics as points at which converging and diverging forces resolve, and from which departures are resisted. In the presence of pervasive noise attractors retain stability, but are nonetheless susceptible to shifts to new attractor positions. Such departures, however, can exceed converging forces, leading to qualitatively new solutions that non-linearly and abruptly differ from the original one. Because the corresponding neural phenomena are physical situated, with its requisite spatiotemporal limitations, attractor solutions are not always achieved, but instead constitute unattained points at the end of a trajectory toward which the attractor is heading. Recently proposed computational models based on brain dynamics, accordingly, accommodate these transient states, where intermediate stages also yield functionally relevant computations [8-10].

In the distributed and highly complex interconnectivity that characterizes global and local influences, attractors thus emerge from a high dimensional state space, where multiple forces shape their trajectory. While the chief factors arise in and through neural effects, these are also modulated by numerous physical factors, like anatomical configuration or impedance resonance, which significantly increase the dimensions of the state space [11]. Linking the theoretical conception of the dynamic event to the physical reality that attractors are intended to model, therefore, is not directly possible [8]; hence, a signature feature having the requisite properties of stability and convergence in the face of extrinsic perturbations is needed; that is, a feature that behaves as a function of state. Of these, the phasic behavior of neural ensembles is the best known and most frequently invoked [11].

Such rhythmic neural activities, generally considered synonymous with oscillations, lend themselves naturally to dynamically stable behavior since rhythmic cycles converge to relatively invariant patterns that can respond to, yet also resist, perturbation. Phasic behavior is a natural outcome of oscillator activity since phase changes describe a cycle whose inner events undergo characteristic and repetitive appearance. Oscillatory activity, moreover, is intrinsically ordered to combinatorial variation, with its coincident phase adjustment to new

${ }^{\star}$ Correspondence to: Denis Larrivee, Loyola University Chicago, USA, E-mail: sallar1@aol.com

Key words: Global brain dynamics, oscillations, desynchronization, stochastic, spike train dependency.

Received: April 02, 2018; Accepted: April 20, 2018; Published: April 23, 2018 
invariant states and the generation of new attractor solutions [12]. Because oscillating activity is scale independent it can physically realize attractor states by combining across inter-areal domains at global as well as local levels, facilitating communication, coordination, and regulation of distributed neural populations that are simultaneously engaged through functional and effective connectivities [13-15].

Oscillatory models for communication posit that synchronization enhances information transmission $[12,16]$, that is, phase alignment tends to facilitate information transfer and behavioral outcome. For enhanced information transfer, therefore, there must be a relationship between oscillator pairs that is characterized by a relatively invariant phase alignment. Synchronization is central, for example, to such theories as Communication through Coherence and Binding by Synchrony that posit that phase consistency optimizes transmission [17]. Conversely, new forms of information transfer require the availability of new portals through which information may be diverted; this is to say that oscillators must be capable of desynchronization in order to access new combinatorial variation. Hence desynchronization is the physical realization of updating to new attractor states [8]. In principle, transference out of the high dimensional attractor basin occurs because the basin no longer constitutes a point of energy stability becoming, instead, increasingly unstable. With sufficient destabilization a critical point is attained that is discretized, a bifurcation, where a new suite of forces balances the attractor configuration; for oscillators destabilization is effected through decoherence.

Accessing and directing new sources of information is a critical feature of global entrainment, which must flexibly respond to varying cues whether of sensory or cognitive origin [18]. Simmons and Barsalou [19], for example, site these cues to modality specific association areas that may combine sensory, motor, or emotion output that, in turn, impact global output. Regulating de-entrainment of oscillator combinations is thus crucial to the realization of behavioral variability. How this is achieved is, as yet uncertain, but is likely to entail basic physical properties of the neural architecture that have been availed for this purpose.

Among factors likely to facilitate these processes, spike timing appears to be a critical, though nonetheless complex, one [11]. Oscillations enhance neuronal spike probability by defining, through repetitive cycles periods of higher excitability, where neurons are sensitive and more responsive to incoming trains, and periods of reduced sensitivity, where spike occurrence is less. By adjusting spike timing it is thus possible to enhance intrinsic tendencies toward synchrony where oscillators can properly align and their frequencies then resonate in unison, or, conversely, to weaken their association leading to decoherence.

Spike discharges at the single neuron level, however, are also highly irregular, described by some measures as Poisson like, probabilistic distributions [20]. Synaptic inputs to cortical neurons measured in vivo, notably, are subject to wide stochastic fluctuation [21]. Tuning curves, in consequence and for example, are broadened, a feature likely to affect phase alignment and desynchronization [22]. Indeed, noise contributions have been shown to significantly alter phase resetting curves [23].

Prevailing mechanisms for regulating bifurcations thus are likely to include deterministic as well as stochastic elements, including pulsing volleys and random spiking that influence spike firing. How these mechanisms are used for the 'good of the organism' that is, the manner by which oscillatory flexibility is mediated and its dependency on the physical background of the neural architecture, and how this enables organismal interactivity, will be the subject of this review.

\section{The state space: Weak coupling and sparse associations}

The need to recombine, that is, to retain variability in oscillator associations, requires that synchronization be of only modest strength, a feature of the state space that constrains how pulsing and stochastic discharges influence oscillator separation. The Theory of Weakly Coupled Oscillators [24] that models synchronization captures this characteristic of modest coupling in the mathematical description given by the Adler equation. For oscillation to persist, weak thus means that interactions lead to phase adjustments without strong perturbations of the oscillatory generative mechanisms. The Adler equation, accordingly, includes terms for divergence, which are ascribed to detuning due to intrinsic frequency differences between oscillator pairs, and for merger, which is determined by the coupling constant and related to the sine function of the phase angle difference. Weak coupling, hence, necessarily includes the presence of both diverging and merging tendencies, the balance between these two opposing factors thereby determining the trajectory undertaken toward synchrony.

Due to the balance between these forces, constancy in the instantaneous phase relation between two oscillators, termed phase locking, is typically never fully achieved [12]; hence, phase precession between coupled oscillators necessarily precesses through all phase angles, with coupling strength continually changing throughout the phase precession cycle. Attractive pull is enhanced when phase tuning is more proximate and reduced when phases are widely separated. In consequence, the fraction of cycle time spent in phase proximity is greater than that when phases are widely separated, a circumstance that is increasingly asymmetric as phase locking values approach a value of 1 . Worded otherwise, phase precession is slowest when phase angles are proximate and fastest when they are distant.

\section{Sparse networks}

Underlying the mechanism of weak coupling described by the Adler equation is a fine structure that is made apparent in mass recordings like the EEG, and is exhibited as a temporal variability in rhythmic neural activity. Termed phase variance, it has been linked to the individual behavior of micro-oscillators [22] that are subject to random spike discharges. Such activity displays considerable 'noise' fluctuation that is related to intraneuronal temporal variation in spike production [23], seen in single neuron current injections, and to variability between groups of neurons that are linked within oscillation circuits. Temporal variation observed in a mass recording like the EEG is thus indicative of a large set of micro oscillators whose alignment is stochastically determined and whose phases are normally distributed about a phase mean. Complicating this conclusion, however, are observations of intermittency in phase alignment. Micro oscillators, in fact, are not simply normally distributed, but, rather, regularly display intermittent episodes of desynchronization, where they no longer align with the oscillating phase [22]. The probability of this desynchronization occurs in inverse relation to its duration, that is, short quick desynchronizations are regularly experienced by all set members at random intervals, whereas longer separations are rare. These observations suggest that at least one effect of noise fluctuation is the generation of a small, independent pocket of micro oscillators that can be desynchronized in preparation for new and novel oscillator combinations.

Unlike models for information transfer that are linked to phase locked states, that is, static and defined by a constancy in instantaneous 
phase relations, the partial synchronization that characterizes weak coupling entails a frequency modulation that alternates during precession between relatively strongly and weakly coupled periods of each cycle. This means also that within the oscillation cycle there exist temporal zones where synchronization is more or less stable, hence, where information transfer is more likely or not to occur. Unlike the fully phase locked state, therefore, where information transfer is postulated to occur only when the instantaneous phase difference is zero, in the weakly coupled model information transfer is likely to be periodic, rather than continuous - that is, to repetitively occur within a portion of each cycle, rather than to occur throughout the period of phase locking - and to occur at less than perfect alignment with the phase of the population oscillator. Indeed, instantaneous frequency modulation appears to be critical to the regulation of phase relations and synchrony $[15,25,26]$. By extension, synchronization and coupling strength also undergo minima repetitively. In other words, within each cycle instabilities are enhanced, making the possibility for desynchronization and bifurcation to another oscillator more likely.

Spike timing influences, accordingly, are framed by the physical circumstances that arise from the weakly coupled state, where spike discharges must adjust to the cyclical reappearance of oscillation instabilities to be effective. On the other hand, the proportion of the cycle occupied by enhanced or weakened coupling can be manipulated by spiking events, which can be expected to modulate oscillator recombination. How can this occur? In the absence of coupling phase precession is a linear function throughout the oscillation cycle [12]. With coupling, however, preferred phase relations that are marked by increased residency time make their appearance. Accordingly, modulating coupling strength can enhance desynchronization when paired with phasic ranges that maximize instability.

\section{Deterministic influences on desynchronization}

Phase resetting tools that employ spike discharges, for example, illustrate how the temporal distribution of oscillation peaks can be modified, either by delaying or advancing them. For instance, charge injection has been used to reset hypersynchronized oscillations that underlie epileptic volleys [27], leading to their desynchronization and relief from epileptic behavior. Analogously, intrinsic spike trains are capable of resetting oscillator phases also. As these are non-random events, therefore, they constitute a deterministic mechanism capable of modifying coupling strength.

The extent of the phase change introduced by pulse trains is typically described by phase resetting curves that relate the phase reset angle to the phase precession angle. A stimulus arriving after $4 / 10$ of the cycle [17], for example, will advance the oscillation phase, while one arriving shortly after the onset of the cycle, within $1 / 10$ of the cycle, will retard it. The time dependency of the phase change is explained in the Wilson Cowan [28] model, which posits that reciprocal volleys of first excitatory and then inhibitory neurons fire to yield the oscillating rhythm. Resetting trains are interpreted in this model to generate a second burst of spiking activity when inhibition effects have sufficiently waned. When pulsing trains occur very early after the onset of the phase cycle, for instance additional excitatory activity postpones inhibitory effects, which then delay the appearance of the next prominent spiking phase.

Such spike trains originate naturally from a variety of cognitive sources. Significantly, current studies now document a number of cue generated, event related potentials that could serve to initiate pulsing trains and influence oscillation phase. Among the most prominent of these is the steady state visual evoked potential (SSVEP) $[29,30]$ that is elicited in response to visual stimuli. Remarkably the SSVEP is responsive not only to a single stimulus but also to trains of stimuli and even to complex imagery, thereby carrying specific, discriminable information content about the cue source. The SSVEP, as well as other event related potentials, are postulated to be stimulus synchronized responses, and so possess the necessary power to emerge as recorded events in the EEG [17], implicating the recruitment of oscillating activity from several sources that thereby enhance power sufficiently to then mediate the phase changes. Outgoing trains from the cued event, for example, appear to function in internal phase resetting in the hippocampus [31] and, importantly for organismal performance, global attentional mechanisms have been shown to control delta phase resetting also [17].

The weak coupling circumstances that define the state space embedding oscillator pairs are critical here for placing constraints on how trains promote dissociation, which is likely to be temporally defined by the periodic reappearance of coupling strength minima. Significantly, pulse trains do not uniformly alter single clocklike oscillators, but rather a distributed population of micro oscillators, which are individually advanced or retarded to greater or lesser degrees; hence, the resulting phase broadening promotes dissociation among microoscillators that fail to align with the main peak.

Weakening synchrony through temporally imposed phase changes has also been shown to influence network activity beyond that of the coupled pair. Through synchronization, for example, embedded oscillators stabilize larger networks [32]. Cross frequency coupling of theta and gamma oscillations, in particular, appears to be critical to the formation of cell assemblies activated during the gamma cycle [33] that are postulated to significantly increase information load. Desynchronization, on the other hand, can be expected to reverse network stabilization to modulate information distribution.

Such information distribution, moreover, can be spatially amplified by introducing distance dependent delays [34]. Since spiking discharges require finite intervals for signal travel, distance dependent delays analogously function like spike trains by broadly spreading phase timing influences. Antiphase states in coupled oscillators [35], incoherent states in all to all coupled oscillator groups, and disorganized states in two dimensional arrays [36] for example, are all consequences of spatial delays. A significant outcome of this form of desynchronization is the induction of wave states. These states are characterized by spreading activity zones known as activity packets that spread across a network [37], where wave speed is determined by cellular and synaptic properties that control the surge of activity. In sparsely coupled networks the introduction of such delays in only a small fraction of connections, for example, is sufficient to yield waves; hence, the emergence of the wave state is a likely outcome of desynchronization, functioning to generate new attractors and to structure novel and variable modes of communication across larger spatial distances.

\section{Stochastic influences on desynchronization}

While pulse trains can deterministically link cue generated potentials to oscillator pairs, the pervasive presence of stochastic fluctuation that characterizes the field of neural activity and defines the background within which oscillations are embedded, is likely to be functionally significant as well. Indeed, evolutionary forces are unlikely to have disregarded its use without costly investment of material and energy reserves [38]. Spiking activities and raster plots reveal, for example, that superimposed on a prominent, but evenly and randomly 
distributed activity background, is a much smaller rhythmic wave [39], which is attributed to noise induced synchrony emerging from spike timing dependencies. Effects on desynchronization and combinatorial variation, accordingly, are likely to reflect the multifaceted, physical properties that characterize the neural 'noise' of the high dimensional cognitive space.

How might noise function in oscillator recombination? Crucially, stochastic processes exhibit system criticality [1], that is, state dependent, phase transitions that mark the appearance of qualitatively new forms of behavior. Local cortical circuits, for example, require a minimal connectivity level for coherent oscillations [40]. Below this critical level synchronous activity is not possible, but once a threshold is achieved oscillations appear, like the rhythmic pattern seen in the raster plots. The presence of these transitions means that the traversal of this critical junction generates a qualitatively different phase where random fluctuations give way to ordered behavior. In a complex system having many degrees of freedom this junction is dynamically and non-linearly determined; hence it can randomly appear at an indefinite number of points. In the case of spiking discharge, this means that the state space is informationally captured by multivariate and multi elicited time courses of spike generation [38], that is, noise driven transitions among multiple and multistable attractors [1] that are physically realized in the spike generating patterns. Indeed large networks of sparsely coupled neurons occur throughout the brain, which, strikingly, are dynamically sensitive to small perturbations [41].

The element of criticality in chaotic behavior thus affords the significant prospect for exploring the full range of rhythmic variation contained within the state space [38]. In principle, therefore, oscillatory variation can influence desynchronization by exposing coupled pairs to a wide variety of input patterns. Its significance, accordingly, lies in its capacity to maximize information exposure, understood as a determination of the maximum Shannon entropy of the system; that is, the information content of the state system is a function of all possible spiking configurations. If needed, the neural system can draw from this content to respond differentially and behaviorally to ongoing environmental change. Hence, chaotic and stochastic outcomes are not irrelevant obstacles to mechanistic and deterministic events but serve a critical role in correctly assessing responsive behavior. Strikingly, comparable conclusions are drawn from machine learning processes that are designated unsupervised [42].

How behaviorally relevant information may be extracted from such information content is an uncertain, but not unresolved computational problem for neural systems. Similar problems are encountered in the case of memory retrieval, where there is a need to distinguish between information capacity and information resolution. Specifically, recognition of unique memory engrams entails a minimization of entropic variation; hence, the system state is required to evolve to a unique representation. In the context of oscillators, for example, discrete phase shifts under the influence of arbitrarily small phase fluctuations can evolve over time to a final state of synchrony [43].

Exploiting these background rhythms to modify entrained oscillatory activity plausibly entails, again, the spike dependency of phasic noise events. An important principle of spike dependency here is that of temporal correlation, better known as Hebb's rule, which is assisted and amplified by oscillations. Spike discharges that follow closely on another discharge, for instance, enhance their connectivities in that direction, a phenomenon that is mediated through their synapses. Conversely, connectivities that are sequentially reversed are weakened [11]. Oscillatory synchronies, for example, establish firing and phasic dependencies; hence, they strengthen coupling through directional persistence. Stochastic firing that may emerge through exploratory criticalities, on the other hand, is temporally independent. This is significant since weakly coupled oscillators traverse all phase angles and widely separated phase intervals are then more likely to be influenced by stochastic events, leading to correspondingly weaker coupling. Accordingly, probabilities for phasic spiking influence from sparse, noise induced rhythms are elevated when coupling strength is least, a circumstance repeated during each oscillation cycle that can facilitate oscillator transfer.

\section{Summary and conclusion}

Organismal behavior entails holistic referencing and the availability of a spectrum of behavioral possibilities that can be autonomously enacted. Cognitive mechanisms underpinning these twin needs emerge from a high dimensional state space, with a multitude of degrees of freedom, assessing environmental variance and structuring often complex responses beneficial to survival. Built from the physical circumstances of an energetically efficient and complexly ordered neural architecture, these mechanisms evoke non-linear dynamical elements that are physically realized in the cyclical and repetitive spiking events of oscillatory activity. Subject to weak coupling constraints oscillator recombination is regulated by complementary deterministic and stochastic events that yield novel forms of information transfer enabling adaptive, reliable, and robust behavior for living systems.

\section{References}

1. Deco G, Jirsa V, Friston KJ (2013) The dynamical and structural basis of brain activity In: Principles of Brain Dynamics: Global State Interactions. Rabinovich MI, Friston KJ, Varona P (eds). Cambridge, MA: MIT Press.

2. Friston K (2013) Free energy and global dynamics. In: Principles of Brain Dynamics Global State Interactions. Rabinovich MI, Friston KJ, Varona P (eds) Cambridge, MA: MIT Press.

3. Kiebel SJ, Friston KJ (2013) Recognition of sequences of sequences using nonlinear dynamical systems. In: Principles of Brain Dynamics: Global State Interactions. Rabinovich MI, Friston KJ, Varona P (eds). Cambridge, MA: MIT Press.

4. Reimann H, Lins J, Schoner G (2015) The dynamics of neural activation variables. $J$. Behav Robot 6: 57-70. [Crossref]

5. Schöner G, Dineva E (2007) Dynamic instabilities as mechanisms for emergence. Dev Sci 10: 69-74. [Crossref]

6. Knips G, Zibner SKJ, Reimann H, Schoner G (2017) A neural dynamic architecture for reaching and grasping integrates perception and movement generation and enables on-line updating. Front Neurorob 11: 9. [Crossref]

7. Schoner G (2009) Development as change of system dynamics: stability, instability, and emergence. In: Toward a Unified Theory of Development. Spencer J, Thomas MSC, McClelland JL (eds) Oxford: Oxford University Press.

8. Rabinovich MK, Varona P (2013) Transient brain dynamics. In: Principles of Brain Dynamics: Global State Interactions. Rabinovich MI, Friston KJ, Varona P (eds). Cambridge, MA: MIT Press. [Crossref]

9. Postlethwaite CM, Dawes HP (2005) Regular and irregular cycling near a heteroclinic network. Nonlinearity 18: 1477-1509. [Crossref]

10. Rabinovich MI, Huerta R, Laurent G (2008) Neuroscience. Transient dynamics for neural processing. Science 321(5885): 48-50. [Crossref]

11. Wang XJ (2010) Neurophysiological and computational principles of cortical rhythms in cognition. Physiol Rev 90: 1195-1268. [Crossref]

12. Lowet E, Roberts MJ, Bonizzi P, Karel J, De Weerd P (2015) Quantifying neural oscillatory synchronization: a comparison between spectral coherence and phaselocking value approaches. PLoS One 11: e0146443. [Crossref]

13. Buzsáki G, Draguhn A (2004) Neuronal oscillations in cortical networks. Science 304: 1926-1929. [Crossref]

14. Buzsaki G (2006) Rhythms of the Brain. New York: Oxford University Press. [Crossref] 
15. Lowet E, Roberts MJ, Peter A, Gips B, DeWeerd P (2017) A quantitative theory of gamma synchronization in macaque V1. eLIFE 6: e26642. [Crossref]

16. Buehlmann A, Deco G (2010) Optimal information transfer in the cortex through synchronization (optimal information transfer). PLoS Comput Biol 6: e1000934. [Crossref]

17. Canavier CC (2015) Phase-resetting as a tool of information transmission. Curr Opin Neurobiol 31: 206-213. [Crossref]

18. Smith L (2009) Stability and flexibility in development. In: Spencer J, Thomas MSC McClelland JL Toward a unified theory of development. Oxford: Oxford University Press. [Crossref]

19. Simmons WK, Barsalou LW (2003) The similarity-in-topography principle: reconciling theories of conceptual deficits. Cog Neuropsychol 20: 451-486. [Crossref]

20. Shadlen MN, Newsome WT (1994) Noise, neural codes and cortical organization. Curr Opin Neurobiol 4: 569-579. [Crossref]

21. Doesburg SM, Green JJ, McDonald JJ, Ward LM (2009) From local inhibition to long-range integration: a functional dissociation of alpha-band synchronization across cortical scales in visuospatial attention. Brain Res 1303: 97-110. [Crossref]

22. Ahn and RubchinskyAhn S, Rubchinsky LL (2013) Short desynchronization episodes prevail in synchronous dynamics of human brain rhythms. Chaos 23: 1-8. [Crossref]

23. Ermentrout GB, Beverlin B 2nd, Troyer T, Netoff TI (2011) The variance of phaseresetting curves. J Comput Neurosci 31: 185-197. [Crossref]

24. Breakspear M, Heitmann S, Daffertshofer A (2010) Generative models of cortical oscillations: neurobiological implications of the kuramoto model. Front Hum Neurosci 4: 190. [Crossref]

25. Atallah BV, Scanziani M (2009) Instantaneous modulation of gamma oscillation frequency by balancing excitation with inhibition. Neuron 62:566-577. [Crossref]

26. Ray S, Maunsell JH. (2010) Differences in gamma frequencies across visual cortex restrict their possible use in computation. Neuron 67: 885-896. [Crossref]

27. Wilson CJ, Beverlin B, Netoff (2011) Chaotic decorrelation of globus pallidus by periodic forcing: a possible mechanism for the therapeutic effects of deep brain stimulation. BMC Neurosci 12(Suppl 1): F3. [Crossref]

28. Wilson HR, Cowan JD (1972) Excitatory and inhibitory interactions in localized populations of model neurons. Biophys $J$ 12: 1-24. [Crossref]

29. Norcia AM, Appelbaum LG, Ales JM, Cottereau BR, Rossion B (2015) The steadystate visual evoked potential in vision research: a review. $J$ Vision 15: 1-46. [Crossref]
30. Yeh CL, Lee PL, Chen WM, Change CY, Wu YT, et al. (2013) Improvement of classification accuracy in a phase-tagged steady-state visual evoked potential based bain computer interface using multiclass support vector machine. BioMed Engin OnLine 12: 46. [Crossref]

31. Jutras MJ, Fries P, Buffalo EA (2013) Oscillatory activity in the monkey hippocampus during visual exploration and memory formation. Proc Natl Acad Sci USA 110: 13144 13149. [Crossref]

32. Acuthan S, Canavier CC (2009) Phase-resetting curves determine synchronization, phase locking, and clustering in networks of neural oscillators. $J$ Neurosci Off J Soc Neurosci 29: 52185233. [Crossref]

33. Watrous AJ, Ekstrom AD (2014) The spectro-contextual encoding and retrieval theory of episodic memory. Front Hum Neurosci 8: 75. [Crossref]

34. Ko TW, Ermentrout GB (2007) Effects of axonal time delay on synchronization and wave formation in sparsely coupled neuronal oscillators. Phys Rev E Stat Nonlin Sof Matter Phys 76: 056206. [Crossref]

35. Schuster HG, Wagner P (1989) Mutual Entrainment of Two Limit Cycle Oscillators with Time Delayed Coupling. Prog Theor Phys 81: 939. [Crossref]

36. Niebur E, Schuster HG, Kammen DM (1991) Collective frequencies and metastability in networks of limit-cycle oscillators with time delay. Phys Rev Lett 67: 2753-2756. [Crossref]

37. Esser SK, Hill SL, Tononi G (2007) Sleep homeostasis and cortical synchronization. I Modeling the effects of synaptic strength on sleep slow waves. Sleep 30: 1617-1630. [Crossref]

38. Rabinovich MI, Abarbanel HD (1998) The role of chaos in neural systems. Neuroscience 87: 5-14. [Crossref]

39. Colgin LL, Denninger T, Fyhn M, Hafting T, Bonnevie T, et al. (2009) Frequency of gamma oscillations routes flow of information in the hippocampus. Nature 462: 353 357. [Crossref]

40. Song S, Sjöström P, Reigl M, Nelson S, Chklovskii D (2005) Highly nonrandom features of synaptic connectivity in local cortical circuits. PLoS Biol 3: e68. [Crossref]

41. Lajoie G, Thivierge JP, Shea-Brown E (2014) Structured chaos shapes spike response noise entropy in balanced neural networks. Front Comput Neurosci 8. [Crossref]

42. Larrivee D (2017) Improving objective assessment in disorders of consciousness: An option for classification technology? Clin Sci Res Reports 1: 1-4. [Crossref]

43. Holzel RW, Krischer K (2013) Pattern recognition minimizes entropy production in a neural network of electrical oscillators. Physics Letters A 377: 2766-2770. [Crossref]

Copyright: $\odot 2018$ Larrivee D. This is an open-access article distributed under the terms of the Creative Commons Attribution License, which permits unrestricted use, distribution, and reproduction in any medium, provided the original author and source are credited. 